\title{
Longitudinal Retinal Changes Induced by Hydroxychloroquine in Eyes without Retinal Toxicity
}

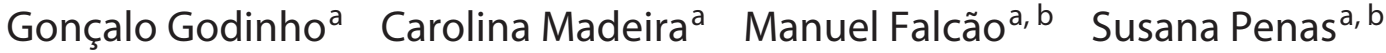 \\ Teresa Dinah-Bragança ${ }^{a}$ Elisete Brandão ${ }^{a}$ Ângela Carneiro ${ }^{a, b}$ \\ Renato Santos-Silva ${ }^{a, b}$ Fernando Falcão-Reis ${ }^{a, b}$ João Beato ${ }^{a, b}$ \\ aDepartment of Ophthalmology, Centro Hospitalar e Universitário São João, Porto, Portugal; ${ }^{\text {b}}$ Department of \\ Surgery and Physiology, Faculty of Medicine of Porto University, Porto, Portugal
}

\section{Keywords}

Ganglion cell layer · Hydroxychloroquine · Optical

coherence tomography $\cdot$ Retina $\cdot$ Retinal thickness

\begin{abstract}
Introduction: This study aimed to evaluate the longitudinal changes in retinal layer thickness in patients treated with hydroxychloroquine without retinal toxicity. Methods: This is a longitudinal retrospective study of patients taking hydroxychloroquine followed in a hydroxychloroquine retinal toxicity screening program of a tertiary hospital between January 2010 and April 2019. Patients who performed 2 optical coherence tomography (OCT) scans at least 1 year apart were included. All subjects with hydroxychloroquine suspected or confirmed retinal toxicity, glaucoma, retinal pathology, or poor segmented images were excluded. Spectral-domain optical coherence tomography (Spectralis HRA-OCT, Heidelberg) was used to evaluate the macular area. Automatically segmented ETDRS retinal thickness maps were compared between the first and the last OCT evaluation available. Full retina (FR), inner retina (IRL), ganglion cells (GCL), inner nuclear (INL), and outer retina (ORL) layer thicknesses were measured in the foveolar, paracentral, and peripheral area.
\end{abstract}

Results: The population included 144 eyes of 144 patients. The mean interval between OCT scans was $38.1 \pm 18.4$ months, and the mean cumulative dose was $406.9 \pm 223.9 \mathrm{~g}$. Foveolar ( $p=0.040, p=0.006$, and $p=0.001$, respectively) and paracentral $(p=0.006, p=0.001$, and $p=0.005$, respectively) FR, IRL, and GCL decreased overtime. No differences were found in INL or ORL. A very weak correlation was found between age and foveal IRL change overtime $(p=0.037 ; R=$ $0.175)$, as well as between the hydroxychloroquine time of use and foveal $\mathrm{GCL}$ variation ( $p=0.032 ; R=0.179$ ). Conclusion: Hydroxychloroquine was found to cause progressive thinning of the inner retinal layers, specifically in the GCL of the foveolar and paracentral areas, but no changes were observed in the outer retina.

(c) 2020 S. Karger AG, Basel

\section{Introduction}

Hydroxychloroquine is an anti-malarial drug commonly used in rheumatologic or dermatological conditions. Its use is partly due to its good safety profile; however, it is estimated that up to $7.5 \%$ of long-term users may develop retinal toxicity [1]. 
The mechanism of drug toxicity is still controversial. Hydroxychloroquine tends to accumulate in pigmented tissues such as retinal pigment epithelium (RPE), inhibiting the activity of anionic transporter 1A2 (OATP1A2) [2]. Another theory suggests that the drug accumulates in the photoreceptors, leading to secondary degeneration of the outer nuclear layer and, subsequently, of RPE [3]. Currently, it is hypothesized that hydroxychloroquine accumulates primarily at the level of the ganglion cell layer but its implications are still unknown [4].

Although usually asymptomatic, retinal toxicity may be associated with photosensitivity and presence of scotomas [5]. Retinal changes include focal disruption of the parafoveal ellipsoid zone, outer retina, and RPE atrophy, being these lesions irreversible and untreatable [6]. Suspension of the drug is mandatory, although these lesions may continue to progress [7].

Screening programs aim for early recognition of unambiguous signs of drug-related retinal toxicity, trying to prevent the irreversible decrease in visual acuity and to enable safe drug use. Several noninvasive diagnostic tests have been used, such as visual fields (VF), multifocal electroretinography (mfERG), autofluorescence (AF), or optical coherence tomography (OCT) [8]. OCT is a sensitive, reproducible, and specific examination that allows qualitative and quantitative assessment of retinal morphology and may detect structural changes that precede functional abnormalities caused by hydroxychloroquine [9].

Based on previous cross-sectional observational studies showing progressive thinning of the inner retinal layers with cumulative drug use in patients taking hydroxychloroquine and no signs of retinal toxicity, this study purposed to evaluate the macular retinal layer thickness changes over time in a population attending the toxicity screening program of a tertiary hospital.

\section{Materials and Methods}

This was a single-center longitudinal retrospective study, performed at the Ophthalmology Department of Centro Hospitalar e Universitário São João, which aimed to evaluate patients referred for retinal toxicity screening associated with hydroxychloroquine treatment between January 2010 and April 2019. This study met the Helsinki ethical criteria. Being a retrospective study involving the collection of unidentified information, the Ethics Committee of Centro Hospitalar e Universitário São João waived the need for approval.

Patients taking or intending to take hydroxychloroquine at our institution are referred for the screening program by their assistant doctor. Then, patients are followed using a standardized protocol that is based on the American Academy of Ophthalmology recommendations reviewed in 2011 and 2016 [8, 10]. Ophthalmic assessments are performed during the first year of therapy to document any structural (AF or OCT) or functional (VF or mfERG) changes, and after 5 years, these tests are repeated annually. Although the mfERG is not a mandatory exam, being this institution a tertiary center with academic motivations, this exam has been included in the protocol.

Patients who performed at least 2 OCT evaluations, with a minimum interval between examinations of 1 year, and taking hydroxychloroquine during that time were included. All patients with suspected or proven retinal toxicity were excluded. Retinal toxicity was confirmed by complementary diagnostic tests according to the following criteria $[8,11]$ : (1) paracentral scotoma proved by the 10-2 Humphrey Field Analyzer program (HFA; Carl Zeiss Meditec, Inc., Dublin, CA, USA); (2) parafoveal hyperautofluorescence; (3) OCT-proven thickness decrease or loss of focal integrity of the parafoveal ellipsoid zone; or (4) decrease in the concentric response of parafoveal rings in mfERG.

Patients with retinal disorders, confirmed or suspected glaucoma, and myopia $>6$ diopters or images with poor segmentation were also excluded. When both eyes met the inclusion criteria, only the right eye was evaluated.

Clinical data were collected from the electronic medical records. Age, sex, weight, manifest refraction, the pathology that led to the prescription of hydroxychloroquine, time and cumulative dose until the first OCT, and time interval and cumulative dose between the first and the last OCT were recorded.

The cumulative dose of hydroxychloroquine was calculated by multiplying the daily dose (in $\mathrm{mg}$ ) by the duration of treatment (in days). When the dose was adjusted during follow-up, the cumulative doses of the various periods were summed. The daily dose of hydroxychloroquine $(\mathrm{mg} / \mathrm{kg})$ was calculated by dividing the daily dose (in $\mathrm{mg}$ ) by the actual weight (in $\mathrm{kg}$ ).

The tomographic evaluation was performed with a spectraldomain OCT (SD-OCT), namely, the Spectralis HRA + OCT 5.1.2.0 system (Heidelberg Engineering, Heidelberg, Germany). The different layers of the macula were automatically segmented, and each segmentation line was manually reviewed. Rarely, manual correction was performed to correct automatic segmentation errors. All images were reviewed by 2 masked doctors (G.G. and C.M.). Retinal thickness maps were obtained in the 9 fovea-centered sectors according to the Early Treatment of Diabetic Retinopathy Study (ETDRS): a central circular area with a diameter of $1 \mathrm{~mm}$, surrounded by 2 concentric rings of 3- and 6-mm diameter (paracentral and peripheral rings, respectively), each with 4 sectors (upper, temporal, inferior, and nasal) (Fig. 1). In each sector, the full retina (FR), inner retina (IRL) (from the inner limiting membrane to the external limiting membrane), outer retina (ORL) (from the external limiting membrane to the RPE), ganglion cell (GCL), and inner nuclear (INL) layer thicknesses were evaluated. The mean of the thicknesses found in the 4 sectors of each concentric ring was also determined [5, 12-14].

Statistical analysis was performed using SPSS software version 23.0. Categorical variables were expressed as frequencies and percentages. Normally distributed variables are represented by mean \pm SD and nonnormal ones by median and interquartile range. The normal distribution of continuous variables was verified by the Shapiro-Wilk test and histogram distribution. Parametric or nonparametric tests were used according to the normality of data. 

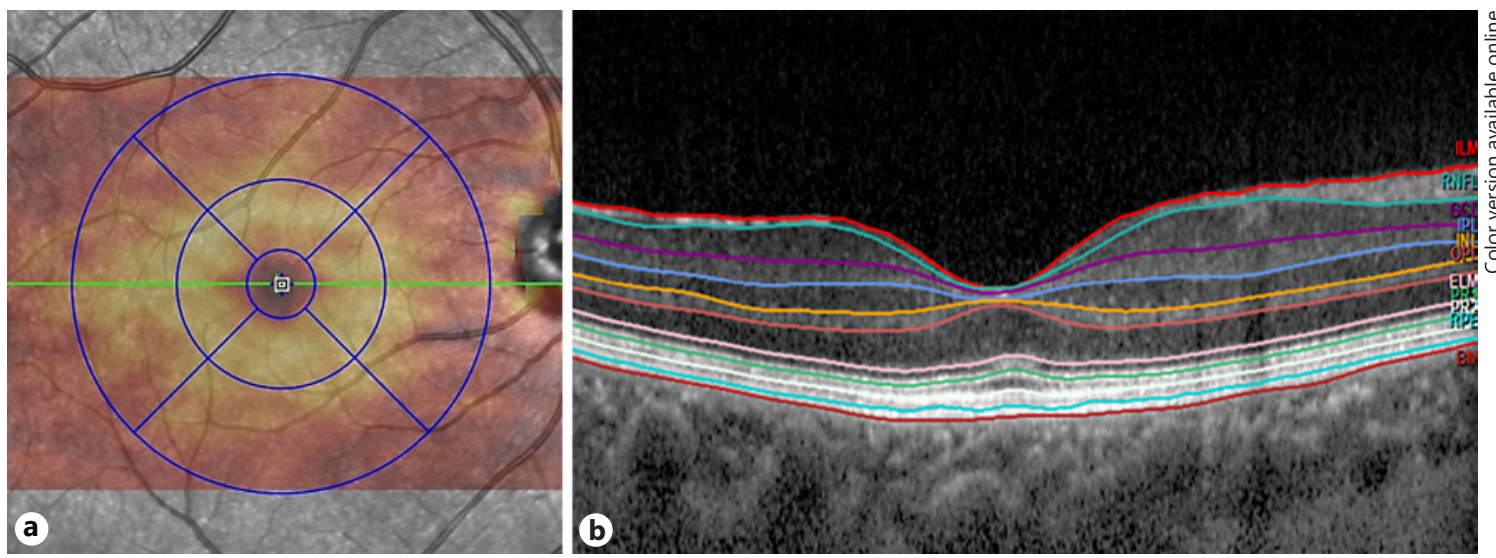

Fig. 1. SD-OCT image: retinal thickness map obtained in the 9 fovea-centered sectors of the ETDRS ring: a central area with a diameter of $1 \mathrm{~mm}$ and 2 concentric rings of diameter 3 and $6 \mathrm{~mm}$ (a); automatic segmentation of the different retinal layers, allowing the assessment of the full retina, ganglion cell layer, inner nuclear layer, and inner and outer retinal layers (b). SD-OCT, spectral-domain optical coherence tomography; ETDRS, Early Treatment of Diabetic Retinopathy Study.

An assessment of the difference in retinal thickness of the different layers between the first and last OCT evaluations was performed, and their correlation with age, gender, weight, disease, time, and cumulative dose of medication was determined by the Spearman test.

\section{Results}

The study included 144 eyes from 144 subjects with a mean age of $53.8 \pm 11.7$ years, and $91.7 \%$ were female. The population had no individuals of Asian descent. Of the patients studied, $49.3 \%$ were taking hydroxychloroquine for systemic lupus erythematosus (SLE), 16.7\% for rheumatoid arthritis, and $10.4 \%$ for Sjogren's syndrome. The remaining cases were diagnosed with nodal osteoarthritis (7.3\%), mixed connective tissue disease (6.3\%), discoid lupus erythematosus (2.8\%), Behçet's disease (2.1\%), and juvenile idiopathic arthritis $(0.7 \%)$.

At the time of the first OCT, patients were being treated with hydroxychloroquine for a median of 4 [2-7] years, and the median cumulative dose was 730 [403.31054.9] g. On average, the time between the 2 evaluations was $38.1 \pm 18.4$ months and the cumulative dose was $407.0 \pm 223.9 \mathrm{~g}$. The daily dose was $4.89 \pm 2.01 \mathrm{mg} / \mathrm{kg}$. The clinical features are described in Table 1 . Neither of the patients presented VF or mfERG with signs of retinal toxicity.

Tables 2 and 3 show the retinal layer thicknesses evaluated. When analyzing FR, there was a decrease in the foveal $(p=0.040)$ and paracentral ring $(p=0.006)$ thick-
Table 1. Demographic and clinical data of the population

\begin{tabular}{lc}
\hline Eyes/patients & $144 / 144$ \\
Age, years & $53.7 \pm 11.6$ \\
Gender (female) & $91.7 \%$ \\
Eye (right) & $88.90 \%$ \\
Visual acuity (logMAR) & $0.0[0.0-0.1]$ \\
Weight, kg & $68.3 \pm 12.3$ \\
Time of use on E1, years & $4[2-7]$ \\
Cumulative dose on E1, g & $730[403.3-1,054.9]$ \\
Daily dose, mg/kg & $4.89 \pm 5.44$ \\
Time between E1 and E2, days & $1,164.9 \pm 566.1$ \\
Dose between E1 and E2, g & $414.3 \pm 229.3$
\end{tabular}

Normal variables are represented by mean \pm SD; nonnormal variables are represented by median [interquartile range]. E1, first evaluation; E2, last evaluation.

ness, but not in the peripheral ring $(p=0.818)$. Regarding the thickness of the IRL, there was a foveal $(p=0.006)$ and paracentral $(p=0.001)$ decrease, but the peripheral ring $(p=0.178)$ had no differences over time. When assessing GCL, there was a decrease in foveal $(p<0.001)$ and paracentral ring ( $p=0.035)$, but not in the peripheral ring $(p=0.065)$. Regarding INL, there was no difference in foveal $(p=0.327)$, paracentral $(p=0.522)$, or peripheral $(p=0.366)$ thickness. Although there was an increase in the nasal ( $p=0.005)$ and superior $(p=0.005)$ paracentral sectors, just as in the nasal peripheral $(p=0,008)$ ORL, there were no differences in the average paracentral $(p=$ 
Table 2. Assessment of FR, IRL, and ORL thickness

\begin{tabular}{|c|c|c|c|}
\hline & $\begin{array}{l}\text { First } \\
\text { examination }\end{array}$ & $\begin{array}{l}\text { Last } \\
\text { examination }\end{array}$ & $p$ value \\
\hline Foveal FR & $277.7 \pm 22.1$ & $275.5 \pm 21.0$ & $0.040^{*}$ \\
\hline \multicolumn{4}{|l|}{ Paracentral FR } \\
\hline Superior & $340.6 \pm 15.9$ & $339.1 \pm 16.0$ & $0.025^{*}$ \\
\hline Nasal & $342.7 \pm 15.3$ & $341.3 \pm 16.1$ & $0.004^{*}$ \\
\hline Inferior & $338.3 \pm 14.4$ & $337.4 \pm 14.3$ & 0.139 \\
\hline Temporal & $327.2 \pm 13.9$ & $323.9 \pm 21.7$ & $0.015^{*}$ \\
\hline Mean & $337.2 \pm 13.8$ & $335.6 \pm 14.8$ & $0.006^{*}$ \\
\hline \multicolumn{4}{|l|}{ Peripheral FR } \\
\hline Superior & $302.8 \pm 14.8$ & $304.4 \pm 14.1$ & $0.030^{*}$ \\
\hline Nasal & $311.9 \pm 14.6$ & $312.1 \pm 15.0$ & 0.685 \\
\hline Inferior & $295.7 \pm 14.1$ & $295.5 \pm 13.7$ & 0.795 \\
\hline Temporal & $280.7 \pm 11.6$ & $279.9 \pm 12.4$ & 0.254 \\
\hline Mean & $297.8 \pm 12.1$ & $297.9 \pm 12.4$ & 0.818 \\
\hline Foveal IRL & $187.3 \pm 22.6$ & $184.8 \pm 20.6$ & $0.006^{*}$ \\
\hline \multicolumn{4}{|c|}{ Paracentral IRL } \\
\hline Superior & $259.7 \pm 15.0$ & $257.8 \pm 14.6$ & $0.010^{*}$ \\
\hline Nasal & $260.8 \pm 14.2$ & $258.5 \pm 15.1$ & $<0.001^{*}$ \\
\hline Inferior & $257.9 \pm 15.0$ & $257.5 \pm 13.6$ & 0.482 \\
\hline Temporal & $246.2 \pm 13.1$ & $244.8 \pm 13.6$ & $0.002 *$ \\
\hline Mean & $256.1 \pm 13.2$ & $254.5 \pm 13.3$ & $0.001^{*}$ \\
\hline \multicolumn{4}{|l|}{ Peripheral IRL } \\
\hline Superior & $224.1 \pm 13.7$ & $225.6 \pm 13.4$ & $0.031^{*}$ \\
\hline Nasal & $234.0 \pm 13.6$ & $233.4 \pm 14.0$ & 0.184 \\
\hline Inferior & $218.6 \pm 13.2$ & $217.9 \pm 12.8$ & 0.387 \\
\hline Temporal & $203.2 \pm 10.8$ & $201.9 \pm 11.1$ & $0.018^{*}$ \\
\hline Mean & $219.9 \pm 11.1$ & $219.1 \pm 12.3$ & 0.178 \\
\hline Foveal ORL & $90.5 \pm 4.8$ & $90.4 \pm 4.7$ & 0.850 \\
\hline \multicolumn{4}{|c|}{ Paracentral ORL } \\
\hline Superior & $80.7 \pm 3.1$ & $81.5 \pm 3.5$ & $0.005^{*}$ \\
\hline Nasal & $82.2 \pm 3.2$ & $83.0 \pm 3.6$ & $0.005^{*}$ \\
\hline Inferior & $80.0 \pm 3.4$ & $80.0 \pm 3.2$ & 0.919 \\
\hline Temporal & $81.0 \pm 3.0$ & $81.3 \pm 3.3$ & 0.312 \\
\hline Mean & $81.0 \pm 2.8$ & $81.4 \pm 3.2$ & 0.068 \\
\hline \multicolumn{4}{|c|}{ Peripheral ORL } \\
\hline Superior & $78.5 \pm 2.9$ & $78.8 \pm 2.8$ & 0.200 \\
\hline Nasal & $77.6 \pm 2.6$ & $78.1 \pm 2.7$ & $0.008^{*}$ \\
\hline Inferior & $76.8 \pm 2.7$ & $77.1 \pm 2.8$ & 0.201 \\
\hline Temporal & $77.5 \pm 2.5$ & $77.7 \pm 2.7$ & 0.368 \\
\hline Mean & $77.6 \pm 2.4$ & $77.9 \pm 2.6$ & 0.086 \\
\hline
\end{tabular}

The paired sample $t$ test (normal variables) was used. Normal variables are represented by mean $\pm \mathrm{SD}$. Values are represented in $\mu \mathrm{m}$. FR, full retina; IRL, inner retinal layer; ORL, outer retinal layer. ${ }^{*} p<0.05$.
Table 3. Assessment of GCL and INL thickness

\begin{tabular}{|c|c|c|c|}
\hline & First examination & Last examination & $p$ value \\
\hline Foveal GCL & $15.0[12.0-18.0]$ & $14.0[12.0-17.0]$ & $0.001^{*}$ \\
\hline \multicolumn{4}{|c|}{ Paracentral GCL } \\
\hline Superior & $52.0[49.0-56.0]$ & $52.0[49.0-55.0]$ & $0.033^{*}$ \\
\hline Nasal & $51.0[48.0-55.0]$ & $50.0[47.0-54.0]$ & $<0.001^{*}$ \\
\hline Inferior & $52.0[49.0-55.0]$ & $52.0[50.0-55.0]$ & 0.804 \\
\hline Temporal & $46.0[43.0-49.0]$ & $46.0[43.0-50.0]$ & 0.376 \\
\hline Mean & $50.3[46.8-53.2]$ & $50.2[47.2-52.7]$ & $0.035^{*}$ \\
\hline \multicolumn{4}{|l|}{ Peripheral GCL } \\
\hline Superior & $37[34.0-39.5]$ & $38.0[35.0-40.2]$ & $0.005^{*}$ \\
\hline Nasal & $37[35.0-40.0]$ & $39.0[36.0-41.0]$ & $<0.001^{*}$ \\
\hline Inferior & $36[33.0-39.0]$ & $37.0[34.0-39.5]$ & 0.575 \\
\hline Temporal & $35[32.0-37.0]$ & $35.0[31.0-37.0]$ & 0.336 \\
\hline Mean & $36.3[34.0-38.5]$ & $36.6[34.9-39.2]$ & 0.065 \\
\hline Foveal INL & $20.0[16.0-24.0]$ & $20.0[16.0-24.0]$ & 0.228 \\
\hline \multicolumn{4}{|l|}{ Paracentral INL } \\
\hline Superior & $41.0[38.0-44.0]$ & $41.0[38.0-44.0]$ & 0.327 \\
\hline Nasal & $41.0[39.0-44.0]$ & $41.0[38.0-44.0]$ & 0.264 \\
\hline Inferior & $40.0[37.0-43.0]$ & $40.0[38.0-43.0]$ & 0.131 \\
\hline Temporal & $38.0[36.0-41.0]$ & $38.0[35.0-40.0]$ & 0.488 \\
\hline Mean & $40.3[38.0-42.3]$ & $39.8[37.5-43.0]$ & 0.522 \\
\hline \multicolumn{4}{|l|}{ Peripheral INL } \\
\hline Superior & $34.0[32.0-36.8]$ & 34.5 [32.0-36.0] & 0.953 \\
\hline Nasal & $34.0[32.0-36.0]$ & $35[33.0-36.0]$ & 0.891 \\
\hline Inferior & $34.0[31.0-36.0]$ & $33[31.0-36.0]$ & 0.937 \\
\hline Temporal & $33.0[32.0-35.0]$ & $33[31.0-35.0]$ & 0.397 \\
\hline Mean & $33.8[32.5-35.3]$ & $33.8[32.2-35.7]$ & 0.366 \\
\hline
\end{tabular}

The Wilcoxon test (nonnormal variables) was used. Nonnormal variables are represented by median [interquartile range]. Values are represented in $\mu \mathrm{m}$. GCL, ganglion cell layer; INL, inner nuclear layer. ${ }^{*} p<0.05$

cumulative dosage of hydroxychloroquine. A very weak correlation ( $p=0.037 ; R=0.175$ ) was found between age and foveal IRL variation over time. Also, a very weak correlation ( $p=0.032 ; R=0.179$ ) was found between the hydroxychloroquine time of use and foveal GCL. Related to the primary diseases of the population, only the foveal FR variation presented a significant difference between rheumatoid arthritis and nodal osteoarthritis $(p=0.010)$.

\section{Discussion/Conclusion}

Hydroxychloroquine use has been increasing globally, and it is estimated that $50 \%$ of SLE patients take it [15]. In this study, SLE was the pathology most identified. Despite its good safety profile, retinal toxicity is an adverse effect to be aware of. AAO [8] lists several major risk fac- 
Table 4. Correlations between mean retinal layer differences and clinical data

\begin{tabular}{lllllll}
\hline & $\begin{array}{l}\text { Foveal } \\
\text { FR }\end{array}$ & $\begin{array}{l}\text { Paracentral } \\
\text { FR }\end{array}$ & $\begin{array}{l}\text { Foveal } \\
\text { IRL }\end{array}$ & $\begin{array}{l}\text { Paracentral } \\
\text { IRL }\end{array}$ & $\begin{array}{l}\text { Foveal } \\
\text { GCL }\end{array}$ & $\begin{array}{l}\text { Paracentral } \\
\text { GCL }\end{array}$ \\
\hline Age & 0.157 & 0.117 & $0.037^{*, 2}$ & 0.137 & 0.246 & 0.675 \\
Gender & 0.115 & 0.292 & 0.187 & 0.918 & 0.289 & 0.261 \\
Weight & 0.657 & 0.147 & 0.946 & 0.273 & 0.413 & 0.202 \\
Disease & $0.040^{*, 3}$ & 0.521 & 0.052 & 0.457 & 0.140 & 0.457 \\
Time between E1 and E2 & 0.547 & 0.954 & 0.560 & 0.131 & $0.032^{*, 4}$ & 0.759 \\
Dosage between E1 and E2 & 0.953 & 0.245 & 0.949 & 0.481 & 0.239 & 0.799 \\
\hline
\end{tabular}

The difference of mean retinal layers that presented significant variations was studied; correlations were found using the Spearman test. FR, full retina; IRL, inner retinal layer; GCL, ganglion cell layer; E1, first evaluation; E2, last evaluation. ${ }^{1}$ Differences between the different diseases were found using the ANOVA test. ${ }^{2}$ The correlation found was very week $(R=0.175){ }^{3}$ The difference found was between rheumatoid arthritis and nodal osteoarthritis $(p=0.012)$, having the first a bigger decrease over time. ${ }^{4}$ The correlation found was very week (Spearman $=$ $0.179) .{ }^{*} p<0.05$.

tors for retinal toxicity, such as daily dose $>5 \mathrm{mg} / \mathrm{kg}$, time of use $>5$ years, renal changes, concomitant use of tamoxifen, and macular diseases. Our population took a daily dose of $4.89 \pm 2.01 \mathrm{mg} / \mathrm{kg}$, which is a value below the recommended upper limit.

Eye screening is critical for early identification of toxicity, allowing drug withdrawal before the development of symptoms. The AAO and Royal College of Ophthalmologists guidelines $[8,11]$ reinforce the need for the use of anatomical and functional tests during the follow-up. The SD-OCT technology enables the identification of photoreceptor layer thickness reduction, ellipsoid zone disruption, and RPE atrophy as specific changes for retinal toxicity [13]. However, these findings may not be very sensitive and the use of quantitative measures could increase the reliability of the screening [7].

Typically, hydroxychloroquine toxicity leads to changes in the outer retina, and its diagnosis is dependent on a multimodal evaluation with anatomical and functional tests. To measure earlier changes, we evaluated patients with no documented toxicity and found a decrease in foveal and paracentral FR thickness. This was accompanied by a concomitant decrease in the IRL and, more specifically, the GCL. These findings are in agreement with other studies $[6,12,14,16-18]$. However, some others deny this possibility $[13,19]$. It is believed that CGL may be the first site with histopathological changes promoted by hydroxychloroquine. Rosenthal et al. [4] described the accumulation of cytoplasmic granules in ganglion cells in animals, with subsequent degeneration and irregular pinocytic nuclei. Changes in the photoreceptor and RPE occurred later. In human studies, these changes were already documented, but also concomitant accumulation in the inner plexiform layer, INL, and RPE and minimal loss of photoreceptors [9].

The decrease in the FR, IRL, and GCL was observed at the foveal and paracentral rings, which is in agreement with changes being initially located at the parafovea, progressing centrifugally [3]. As so, these findings may suggest that these changes can begin at the GCL because in these areas these cells are denser and disposed of in several layers, unlike the remaining retina [20].

We found no changes in the average ORL. These patients had no known toxicity and, consequently, no ORL atrophy developed. It also showed that changes in the IRL happen before changes in the ORL. However, we found zones of sectoral increase in the ORL. Hydroxychloroquine affects $\mathrm{pH}$ and lysosome function, leading to a decrease in RPE function, autophagy, and consequent decrease in the phagocytosis of external photoreceptors [21]. At an early stage, this functional disruption may lead to an increase in the ORL thickness.

Related to the mean retinal layer changes, no relevant correlations were found with sex, weight, and cumulative dosage of hydroxychloroquine. However, a very weak correlation was found between foveal GCL change and the time between the 2 examinations. Otherwise, only a very weak correlation was found between age and foveal IRL variation. Other than that, rheumatoid arthritis presented a bigger difference in foveal FR only in comparison with nodal osteoarthritis. Given the strength of the correlations, these findings do not seem to be enough robust 
to make other conclusions. However, these correlations were only related to the foveal area, not with the paracentral area, which could indicate that changes in the paracentral area are more sensitive to hydroxychloroquine use than the foveal area. In the paracentral area, we can speculate that these changes are dose and time independent.

This study demonstrates that there are changes in the inner retina while taking hydroxychloroquine. As retinal histology cannot be evaluated in vivo, OCT allows a histopathological evaluation not possible otherwise. However, it is important to know whether these changes represent true retinal toxicity or a consequence of chronic use, as the first histopathological changes may occur 1 week after drug initiation [20].

Neither of the patients had retinal toxicity signs in functional examinations: mfERG and/or visual fields. As so, we may extrapolate that there is no correlation between the structural findings seen and the functional hydroxychloroquine criterion currently used. This assumption could mean that structural findings are prior to functional changes or that current functional examinations may not detect these subtle changes. Further studies are necessary to confirm it. Moreover, because of the small magnitude of the values, it is important to know if it is clinically feasible to take information from these findings.

Compared to others, this work has several advantages. It is a longitudinal study, allowing us to evaluate changes over time. Also, it presents a large population sample.

However, it is a retrospective study and it is not possible to control the moments of evaluation. Also, we assume compliance with therapy based on patients' reports. Besides that, it is a heterogeneous sample concerning the doses made and the timing of evaluation. Other than that, it lacks a group control of healthy patients, which is attenuated by the lack or very weak strength of the correla- tions found in our study related to age, sex, and primary disease. Despite the large population sample, we must acknowledge that several hypotheses were tested, which can limit the interpretation of the results.

In conclusion, there was a significant decrease in the foveolar and paracentral inner retinal layers in patients taking hydroxychloroquine without retinal toxicity. This decrease was accompanied by a reduction in the CGL, where the first signs of the therapy may manifest.

\section{Statement of Ethics}

This study was performed in compliance with the Helsinki ethical criteria. Ethics Committee of Centro Hospitalar e Universitário São João waived the need for approval for this study. Written informed consent was obtained.

\section{Conflict of Interest Statement}

The authors have no conflicts of interest to declare.

\section{Funding Sources}

The authors declare no funding support.

\section{Author Contributions}

All authors whose names appear on the submission (1) made substantial contributions to the conception or design of the work; or the acquisition, analysis, or interpretation of data; (2) drafted the work or revised it critically for important intellectual content; (3) approved the version to be published; and (4) agree to be accountable for all aspects of the work in ensuring that questions related to the accuracy or integrity of any part of the work are appropriately investigated and resolved.

\section{References}

1 Melles RB, Marmor MF. The risk of toxic retinopathy in patients on long-term hydroxychloroquine therapy. JAMA Ophthalmol. 2014;132(12):1453-60.

2 Xu C, Zhu L, Chan T, Lu X, Shen W, Madigan $\mathrm{MC}$, et al. Chloroquine and hydroxychloroquine are novel inhibitors of human organic anion transporting polypeptide 1A2. J Pharm Sci. 2016;105(2):884-90.

3 Marmor MF. Comparison of screening procedures in hydroxychloroquine toxicity. Arch Ophthalmol. 2012;130(4):461-9.
4 Rosenthal AR, Kolb H, Bergsma D, Huxsoll D, Hopkins JL. Chloroquine retinopathy in the rhesus monkey. Invest Ophthalmol Vis Sci. 1978;17(12):1158-75.

5 Latasiewicz M, Gourier H, Yusuf IH, Luqmani R, Sharma SM, Downes SM. Hydroxychloroquine retinopathy: an emerging problem. Eye. 2017;31(6):972-6.

6 Pasadhika S, Fishman GA, Choi D, Shahidi M. Selective thinning of the perifoveal inner retina as an early sign of hydroxychloroquine retinal toxicity. Eye. 2010;24(5):756-63.
7 Allahdina AM, Stetson PF, Vitale S, Wong WT, Chew EY, Iii FLF, et al. Optical coherence tomography minimum intensity as an objective measure for the detection of hydroxychloroquine toxicity. Invest Ophthalmol Vis Sci. 2018;59(5):1953-63.

8 Marmor MF, Kellner U, Lai TY, Melles RB, Mieler WF. Recommendations on screening for chloroquine and hydroxychloroquine retinopathy (2016 Revision). Ophthalmology. 2016;123(6):1386-94. 
9 Yülek F, Uğurlu N, Akçay E, Kocamış Sİ, Gerçeker S, Erten Ş, et al. Early retinal and retinal nerve fiber layer effects of hydroxychloroquine: a follow up study by sdOCT. Cutan Ocul Toxicol. 2013;32(3):204-9.

10 Marmor MF, Kellner U, Lai TY, Lyons JS, Mieler WF. Revised recommendations on screening for chloroquine and hydroxychloroquine retinopathy. Ophthalmology. 2011; 118(2):415-22.

11 Fielder A, Graham E, Jones S, Silman A, Tullo A. Royal college of ophthalmologists guidelines: ocular toxicity and hydroxychloroquine. Eye. 1998;12(Pt 6):907-9.

12 Sisternes L, Hu J, Rubin D, Marmor M. Analysis of inner and outer retinal thickness in patients using hydroxychloroquine prior to development of retinopathy. JAMA Ophthalmol. 2016;134:511-9.
13 Modi YS, Au A, Parikh VS, Ehlers JP, Schachat AP, Singh RP. Volumetric single-layer inner retinal analysis in patients with hydroxychloroquine toxicity. Retina. 2016; 36(10): 1941-50.

14 Gil P, Nunes A, Farinha C, Teixeira D, Pires I, Silva R. Structural and functional characterization of the retinal effects of hydroxychloroquine treatment in healthy eyes. Ophthalmologica. 2019;241(4):226-33.

15 Scholl HP, Shah SM. We need to be better prepared for hydroxychloroquine retinopathy. JAMA Ophthalmol. 2014;132(12):1460-1.

16 Uslu H, Gurler B, Yildirim A, Tatar MG, Aylin Kantarc1 F, Goker H, et al. Effect of hydroxychloroquine on the retinal layers: a quantitative evaluation with spectral-domain optical coherence tomography. JAMA Ophthalmol. 2016;2016:8643174-8.

17 Ulviye Y, Betul T, Nur TH, Selda C. Spectral domain optical coherence tomography for early detection of retinal alterations in patients using hydroxychloroquine. Indian J Ophthalmol. 2013;61(4):168-71.
18 Bulut M, Erol MK, Toslak D, Akidan M, Kaya Başar E, Çay HF. A new objective parameter in hydroxychloroquine-induced retinal toxicity screening test: macular retinal ganglion cell-inner plexiform layer thickness. Arch Rheumatol. 2018;33(1):52-8.

19 Lee MG, Kim SJ, Ham DI, Kang SW, Kee C, Lee J, et al. Macular retinal ganglion cell-inner plexiform layer thickness in patients on hydroxychloroquine therapy. Invest Ophthalmol Vis Sci. 2014;56(1):396-402.

20 Kan E, Yakar K, Demirag MD, Gok M. Macular ganglion cell-inner plexiform layer thickness for detection of early retinal toxicity of hydroxychloroquine. Int Ophthalmol. 2018; 38(4):1635-40.

21 Yusuf IH, Sharma S, Luqmani R, Downes SM. Hydroxychloroquine retinopathy. Eye. 2017; 31(6):828-45. 